\title{
A proteome-wide screen identifies valosin-containing protein as an essential regulator of podocyte endoplasmic reticulum stress
}

\author{
HUANG ZhiYong ${ }^{1 \dagger}$, HONG Quan ${ }^{1 \dagger}$, XUE Peng $^{2}$, PAUL Goulding ${ }^{3}$, FENG Zhe ${ }^{1}$, \\ WANG LiYuan ${ }^{1}$, MEI Yan ${ }^{1}$, WU LingLing ${ }^{1}$, CHEN XiangMei ${ }^{1 *} \&$ WU Di ${ }^{1 *}$ \\ ${ }^{1}$ State Key Laboratory of Kidney Disease (2011DAV00088), Kidney Department \& Institute of Nephrology, Division of Clinical Internal Medicine, \\ Chinese People's Liberation Army General Hospital, Beijing 100853, China; \\ ${ }^{2}$ Laboratory of Proteomics, Institute of Biophysics, Chinese Academy of Sciences, Beijing 100101, China; \\ ${ }^{3}$ Nonlinear Dynamics Ltd, Keel House, Garth Heads, Newcastle upon Tyne, NE1 2JE, UK
}

Received April 13, 2012; accepted April 24, 2012; published online May 30, 2012

\begin{abstract}
To investigate proteins expressed in the renal tissue of the passive Heymann nephritis (pHN) rat model, we prepared pHN rat models with anti-FxA1 serum and analyzed the proteins differentially expressed in the kidney tissue with label-free liquid chromatography-tandem mass spectrometry. We then analyzed in depth the endoplasmic reticulum stress (ERS)-related protein using an online bioinformatics platform. Forty-one differential proteins and their annotations were obtained. Gene Ontology (GO) function analysis showed that 16 proteins were involved in cellular metabolism and 22 were proteins related to catalytic activity, including protein folding or ATPase. Protein-GO networks indicated that VCP could interact with the ERS marker HSPa5, with both involved in a single pathway. On inhibition of podocyte VCP by RNAi under normal conditions, the HSPa5 expression level did not change, but when the cell was subjected to ERS by tunicamycin, HSPa5 expression significantly increased with RNAi of VCP when compared with the tunicamycin-treated group. Our results showed that ERS plays an important role in podocyte injury of membranous nephropathy and is mediated by an HSPa5-VCP signaling pathway, in which the most predominant proteins are those related to cellular metabolism and catalytic activity.
\end{abstract}

passive Heymann nephritis, ER stress, valosin-containing protein, proteomic

Citation: Huang Z Y, Hong Q, Xue P, et al. A proteome-wide screen identifies valosin-containing protein as an essential regulator of podocyte endoplasmic reticulum stress. Chin Sci Bull, 2012, 57: 2493-2505, doi: 10.1007/s11434-012-5250-8

Membranous nephropathy (MN), a common nephrotic syndrome in adults, is an important cause of chronic kidney disease $[1,2]$. The distinctive pathologic feature of $\mathrm{MN}$ is the presence of immune deposits beneath podocytes [3]. The mechanism of podocyte damage is thought to be quite complex, involving activation of complement and cytokines, oxidative stress, and autophagy. The passive Heymann nephritis (pHN) model is the most commonly used animal model to simulate the conditions of MN [4,5]. Although

\footnotetext{
$\dagger$ These authors contributed equally to this work.

*Corresponding authors (email: wudi@301hospital.com.cn; xmchen301@126.com) Recommended by Prof. ZHANG Xue (Editorial Board Member)
}

previous studies have used the $\mathrm{pHN}$ model to analyze the mechanism of MN development at multiple sites, few have focused on the gene changes of the entire kidney. The current study therefore analyzed differentially expressed proteins of the $\mathrm{pHN}$ rat model with high-throughput technology.

In the past, mass spectrometry (MS) has been of little use in quantifying differential proteins, being limited to mainly qualitative research. Recently, however, comparative quantification of label-free liquid chromatography (LC)-MS proteomics data has emerged as an alternative approach [6]. Label-free quantification [7] MS can determine the differential expression of proteins. In this method, proteins are compared based on the intensities of extracted ion chroma- 
tograms from complex samples such as enzymatic digests. This method does not require metabolic, chemical, or enzymatic labeling or premixing of the samples [8]. Multiple data sets, typically run in triplicate, are aligned using mass and LC elution time, which improves the sensitivity and accuracy of results when compared with the traditional twodimensional technology [9-11]. Therefore, label-free quantification MS has the advantages of convenience and reliable protein quantification and identification.

This study on the pHN rat model used label-free quantitative MS for high-throughput identification of differentially expressed proteins at several time points. The endoplasmic reticulum stress (ERS)-related protein was then analyzed in depth using the online analysis platform found at http:// www.uniprot.org and http://bioinfo.capitalbio.com/mas3.0/.

\section{Materials and methods}

\subsection{Experimental animals}

Nine 180-200 g male SD (Sprague-Dawley) rats were purchased from Beijing Huafukang Company and divided into three groups containing three animals each: control, $14 \mathrm{~d}$ postinjection, and $21 \mathrm{~d}$ postinjection. Experimental animals were housed under specific pathogen-free conditions: $22 \pm$ $1{ }^{\circ} \mathrm{C}, 40 \%$ humidity, 12-h:12-h light/dark cycle, and free access diet. The study was approved by the Institutional Animal Care and Use Committee at the Chinese PLA General Hospital and Military Medical Postgraduate College.

\subsection{Reagents}

Reagents used were acetonitrile (AN; Fisher Scientific, Waltham, MA, USA), formic acid (FA; Fisher Scientific), dithiothreitol (DTT; Sigma, St. Louis, MO, USA), iodoacetemide (IAA; Amresco, Solon, $\mathrm{OH}, \mathrm{USA}$ ), $\mathrm{NH}_{4} \mathrm{HCO}_{3}$ (Sigma), trypsin enzymolysis liquid (Promega, Madison, WI, USA), BCA protein concentration detection reagent kit (Pierce, Rockford, IL, USA), tunicamycin (Tm; Sigma), rabbit polyclonal anti-HSPa5, VCP antibody (Santa Cruz Biotechnology, Santa Cruz, CA, USA), and anti-rabbit IgG (Santa Cruz Biotechnology).

\subsection{Preparation and confirmation of PHN model}

Tubular brush border extracts and anti-Fx1A serum was prepared according to the literature [12-14]. Antisera at $1 \mathrm{~mL} / 100 \mathrm{~g}$ (titer $1: 250$ ) were given as an initial tail vein injection, followed by a second dose $1 \mathrm{~h}$ later and one further booster injection. The control group received intravenous injection of the same dose of saline. All pHN rats had heavy proteinuria (urine protein $>100 \mathrm{mg} / \mathrm{d}$; normal <20). At 14 and $21 \mathrm{~d}$ after injection, rats were anesthetized by intraperitoneal injection of $2 \%$ sodium pentobarbital (40 $\mathrm{mg} / \mathrm{kg}$ ) prior to collection of kidney tissue samples.

\subsection{Cell culture and siRNA transfection}

Preserved mouse podocytes (MPCs) provided by Dr. Peter Mundel (University of Miami, Miami, FL, USA) were cultured as described previously [15]. Differentiated podocytes were treated with $2.5 \mathrm{ng} / \mathrm{mL}$ Tm (Sigma) for $10 \mathrm{~h}$ to induce the ERS.

siRNA-targeting VCP was purchased from Santa Cruz Biotechnology (cat. sc-37188). The siRNA was transfected into MPCs by Jetprime Polyplus transfection reagent (Polyplus-transfection Inc., New York, NY, USA) according to the manufacturers' recommendations.

\subsection{Protein preparation}

Kidney tissues were lysed in buffer solution $(50 \mathrm{mmol} / \mathrm{L}$ Tris- $\mathrm{HCl}$ ( $\mathrm{pH} 7.6$ ), 150 mmol/L NaCl, 1\% NP-40, 0.1\% sodium dodecyl sulfate (SDS), $0.5 \%$ deoxycholic acid, 1 $\mu \mathrm{g} / \mathrm{mL}$ leupeptin, $1 \mu \mathrm{g} / \mathrm{mL}$ aprotinin, and $0.5 \mathrm{mmol} / \mathrm{L}$ phenylmethylsulfonyl fluoride), then cooled on ice for $30 \mathrm{~min}$ prior to centrifuging at $12000 \mathrm{r} / \mathrm{min}$ for $30 \mathrm{~min}$ at $4^{\circ} \mathrm{C}$. The supernatant was removed and the protein concentration was determined by the BCA method (Bio-Rad, Hercules, CA, USA). Then $10 \mathrm{mmol} / \mathrm{L}$ DTT was incubated with $100 \mu \mathrm{g}$ protein at $56^{\circ} \mathrm{C}$ for $1 \mathrm{~h}$. After cooling to room temperature, the protein was alkalized by $50 \mathrm{mmol} / \mathrm{L}$ IAM in darkness for $45 \mathrm{~min}$. Excess IAM was removed with $40 \mathrm{mmol} / \mathrm{L}$ DTT at room temperature. The sample was further diluted five times with $25 \mathrm{mmol} / \mathrm{L} \mathrm{NH}_{4} \mathrm{HCO}_{3}$. Trypsin $1 \%$ was added to digest the protein for 12 and $6 \mathrm{~h}$ at $37^{\circ} \mathrm{C}$. FA $0.1 \%$ was used to terminate digestion, and the debris was removed by centrifugation at $13000 \mathrm{r} / \mathrm{min}$ for $10 \mathrm{~min}$. The supernatant was stored at $-80^{\circ} \mathrm{C}$ for $\mathrm{MS}$ analysis.

\subsection{LC-MS analysis}

The multidimensional protein identification system is composed of an LTQ-ion trap (ThermoFisher Scientific, Waltham, USA) mass spectrometer with an electrospray ionization (nano-ESI) source. We have performed LC-MS analysis previously [16]. Briefly, $100 \mu \mathrm{g}$ of digested peptides were pre-fractionated then were injected onto a self-made biphasic capillary column by high-pressure nitrogen. The mobile phase consists of three solutions: A (5\% AN, $0.1 \%$ FA), B ( $80 \%$ AN, $0.1 \%$ FA), and C ( $800 \mathrm{mmol} / \mathrm{L}$ ammonium acetate, $5 \%$ AN, $0.1 \%$ FA). Solution A was used to desalt the biphasic capillary column for $45 \mathrm{~min}$, after which solution $\mathrm{C}$ was used to wash it stepwise, with the concentration of ammonium acetate increasing from 0 to $800 \mathrm{mmol} / \mathrm{L}$. The elution from the column was loaded onto a C18 analytical capillary chromatography column and a fraction from the column was ionized by electrospray for MS analysis. The spray voltage was set to $2.0 \mathrm{kV}$, and the temperature of the heated capillary was set to $200^{\circ} \mathrm{C}$. MS data were collected in a data-dependent acquisition mode. Parameters 
related to MS/MS data acquisition were set as follows: normalized collision energy $35 \%$, ion selection threshold 200 counts, activation Q 0.250 , activation time $30 \mathrm{~ms}$, and dynamic exclusion time $30 \mathrm{~s}$. The elution gradient of HPLC and MS scanning were controlled by XCalibur (Fisher Scientific).

\subsection{Data processing and protein identification}

LC-MS data were processed using Progenesis LC-MS software (Nonlinear Dynamics Corp., Newcastle upon Tyne, UK) using the default settings. Proteins were identified using the NCBI rat database (March 2011). The GI number of proteins was transformed into UniProtKB on http://www.uniprot. org [17]. Pathway networks and protein functional grouping were generated by the online platform at http://bioinfo.capitalbio.com $/ \mathrm{mas} 3.0 /$.

\subsection{Western blotting}

Fifty micrograms of total protein was separated by $7.5 \%$ SDS-PAGE, transferred to a membrane, and blocked with $5 \%$ skim milk, probed with primary antibody for $2 \mathrm{~h}$ at room temperature, and incubated with horseradish peroxidase-conjugated secondary antibody. $\beta$-actin was used for the sample control. Immunoreactive bands were visualized using an enhanced chemiluminescent system.

\subsection{Real-time PCR assay}

RNA was extracted from tissues and cells using TRIzol reagent (Invitrogen, Carlsbad, CA, USA) and was reverse transcribed to cDNA using M-MLV reverse transcriptase $(2 \mu \mathrm{g})$ (Invitrogen). The cDNA was used as a template in quantitative real-time PCR reactions performed using TaqMan PCR Master Mix and an ICycler system (Bio-Rad). The following primers and probe were designed from the full-length $V C P$ mRNA sequence and synthesized by SBS Biotechnology Corp. (Beijing, China): sense, 5'-AAACCGTGGTAGAGGTGCCA-3'; antisense, 5'-CTTGGAAGGTGTCATGCCAA-3'; and TaqMan probe, 5'-FAMCAGTATCCTGTGGAGCACCCAGACAAATTCTAMRA-3'. As an internal control, rat $G A P D H$ was amplified using the following: sense, 5'-GGCATGGACTGTGGTCATGAG-3'; antisense, 5'-TGCACCACCAACTGCTTAGC-3'; and TaqMan probe, FAM-5'-CCTGGCCAAGGTCATCCATGACAACTTTAMRA-3'. Relative expression (fold-change vs. control) was quantified by the $2^{-\Delta \Delta C_{\mathrm{t}}}$ method [18].

\subsection{Statistical processing}

All data were analyzed using SPSS 13.0 (SPSS Inc., Chicago, IL, USA) software; data are expressed as the mean \pm $\mathrm{SD}$. The differences between the two groups were compared with ANOVA, and $P<0.05$ was considered statistically significant.

\section{Results}

\subsection{LC-MS analysis results}

There were 2134 total proteins and 234 differentially expressed proteins in all three experimental groups. Of those, 41 had $20>$ NRatio $>1.5$ (upregulation) or $0.05<$ NRatio $<$ 0.66 (downregulation) (NRatio: the change ratio of the up/ down-regulated protein on day 14 or 21 versus control). Five proteins have not been annotated in the Uniprot database, including rCG25753 isoform CRA_b, rCG53940 isoform CRA_a, aldolase B isoform CRA_a, rCG55135 isoform CRA_c, and alanine-glyoxylate aminotransferase 2 isoform CRA_c, and 36 proteins have the corresponding Uniprot ID. Table 1 shows how the proteins were grouped.

\subsection{Classification of differentially expressed proteins}

All differentially expressed proteins, upregulated or downregulated at day 14 or 21 , were classified into three groups, A, B, and C. Proteins in group A were downregulated at day 14 , but upregulated at day 21; group B showed downregulation at days 14 and 21; and group $\mathrm{C}$ showed upregulation at days 14 and 21. As shown in Figure 1, group A contained UK114, B4F768, CALB1, THIM, OAT, COTL1, Q68FT4, ACTN4, DECR, D3ZZN3, Q66HT1, Q63011, ACON, Q3MHS9, HSP7C, ADT2, Q6AYS2, and Q7TMC7; group B contained DCXR, GSH0, GRP75, Q9QYA6, ATPB, XYLB, G3V826, GRP78g and GSH1; and group C contained NIT2, IDHC, AATC, Q7TP54, TERA, ST1C2, D4A2K1, AASS, and GSHR.

\subsection{Gene Ontology analysis of differentially expressed proteins}

Differentially expressed proteins were analyzed according to the Gene Ontology (GO) classification. As shown in Figure 2(a), among these proteins, 23 contributed to cellular structure, 30 to molecular function, and 22 to biological processes. The subgroup analysis is shown in Figure 2(b)(d). In the biological process category, proteins related to cellular processes have the highest incidence with 19 proteins. In this subgroup, 16 proteins are involved in cellular metabolic processes, as shown in Table 2. Other biological processes included those of multicellular organisms, e.g. biological regulation and response to stimuli. The proteins related to cell structure include those needed to build, e.g. organelles and macromolecules. Of the proteins related to molecular function, most are related to catalytic activity (22 proteins); details are listed in Table 3. Other proteins are related to binding or oxidoreductase activity function. 
Table 1 Protein annotation of PHN model

\begin{tabular}{|c|c|c|c|c|c|c|c|}
\hline \multirow{2}{*}{ Cat. } & \multirow{2}{*}{ Accession } & \multirow{2}{*}{ Uniprot AC } & \multirow{2}{*}{ Protein name } & \multirow{2}{*}{ Gene name } & \multirow{2}{*}{ Description } & \multicolumn{2}{|c|}{ Folder change ratio } \\
\hline & & & & & & $14 \mathrm{~d} / \mathrm{N}$ & $21 \mathrm{~d} / \mathrm{N}$ \\
\hline \multirow{18}{*}{ A } & gi|4456766 & P52759 & UK114 & Hrsp12, Psp1 & perchloric acid soluble protein & 0.0074 & 0.0108 \\
\hline & gi|195540087 & B4F768 & B4F768 & Aldh4al LOC641316 & Aldh4a1 protein & 0.2834 & 0.7630 \\
\hline & gi|203237 & P07171 & CALB1 & Calb1 & calbindin-d28k & 0.3257 & 0.2984 \\
\hline & gi|18426866 & P13437 & THIM & Acaa2 & 3-ketoacyl-CoA thiolase, mitochondrial & 0.3699 & 0.4622 \\
\hline & gi|11968102 & P04182 & OAT & Oat & ornithine aminotransferase, mitochondrial precursor & 0.4172 & 0.5379 \\
\hline & gi|157823483 & B0BNA5 & COTL1 & Cotll, Clp & coactosin-like protein & 0.4255 & 0.6209 \\
\hline & gi|51260799 & Q68FT4 & Q68FT4 & Suclg2 & Suclg2 protein & 0.4286 & 0.4441 \\
\hline & gi|6636119 & Q9QXQ0 & ACTN4 & Actn 4 & alpha-actinin 4 & 0.4337 & 0.5823 \\
\hline & gi|37748456 & Q64591 & DECR & Decrl, Decr & 2,4-dienoyl CoA reductase 1 , mitochondrial & 0.4360 & 0.5160 \\
\hline & gi|157818027 & D3ZZN3 & D3ZZN3 & Acss1, rCG_37494 & acetyl-coenzyme A synthetase 2-like, mitochondrial & 0.4393 & 0.5023 \\
\hline & gi|158081751 & Q66HT1 & Q66HT1 & Aldob, rCG_60366 & fructose-bisphosphate aldolase B & 0.4505 & 0.8098 \\
\hline & gi|802111 & Q63011 & Q63011 & MGC72973 & zero beta-globin & 0.4773 & 0.6468 \\
\hline & gi|40538860 & Q9ER34 & $\mathrm{ACON}$ & Aco 2 & aconitate hydratase, mitochondrial precursor & 0.4863 & 0.6475 \\
\hline & gi|76253725 & Q3MHS9 & Q3MHS9 & Cct6a,rCG_21714 & T-complex protein 1 subunit zeta & 0.4927 & 0.4606 \\
\hline & gi|13242237 & P63018 & HSP7C & Hspa8, Hsc70 Hsc73 & heat shock cognate $71 \mathrm{kD}$ protein & 0.5872 & 0.3419 \\
\hline & gi|32189350 & Q09073 & ADT2 & Slc25a5, Ant 2 & ADP/ATP translocase 2 & 0.4028 & 0.4346 \\
\hline & gi|58865994 & Q6AYS2 & Q6AYS2 & Sfxn1, $r C G \_24191$ & sideroflexin-1 & 0.4429 & 0.4360 \\
\hline & gi|33086606 & Q7TMC7 & Q7TMC7 & Tf Srprb & Ab2-417 & 0.7389 & 0.4420 \\
\hline \multirow{14}{*}{ B } & gi|149019103 & - & - & - & rCG25753, isoform CRA_b & 0.7333 & 1.6667 \\
\hline & gi|19705501 & Q920P0 & DCXR & $D c x r, G l b$ & $L$-xylulose reductase & 0.9750 & 2.0833 \\
\hline & gi|8393446 & P48508 & GSH0 & Gclm, Glclr & glutamate—cysteine ligase regulatory subunit & 0.9856 & 1.5379 \\
\hline & gi|1000439 & P48721 & GRP75 & Hspa9, Grp 75 Hspa9a & grp75 & 0.9776 & 1.6098 \\
\hline & gi|6523793 & Q9QYA6 & Q9QYA6 & $S l c 4 a 4, n b c$ & electrogenic $\mathrm{Na}^{+}$bicarbonate cotransporter & 0.9900 & 1.5812 \\
\hline & gi|203033 & P10719 & ATPB & Atp $5 b$ & F1-ATPase beta subunit & 1.2185 & 1.7899 \\
\hline & gi|76096348 & Q3MIF4 & XYLB & Xylb & xylulose kinase & 1.3200 & 2.4300 \\
\hline & gi|149034221 & G3V826 & G3V826 & $T k t, r C G_{-} 42377$ & transketolase, isoform CRA_a & 1.4193 & 1.5625 \\
\hline & gi|149055818 & - & - & - & rCG53940, isoform CRA_a & 1.4390 & 1.7236 \\
\hline & gi|149020188 & - & - & - & aldolase B, isoform CRA_a & 1.4711 & 2.3388 \\
\hline & gi|149045753 & - & - & - & rCG55135, isoform CRA_c & 1.5639 & 2.1504 \\
\hline & gi $\mid 25742763$ & P06761 & GRP78 & Hspa5, Grp78 & $78 \mathrm{kD}$ glucose-regulated protein precursor & 1.5961 & 2.3973 \\
\hline & gi $\mid 149027328$ & - & - & - & alanine-glyoxylate aminotransferase 2 , isoform CRA_c & 1.6021 & 2.4187 \\
\hline & gi|25742748 & P19468 & GSH1 & Gclc, Glclc & glutamate - cysteine ligase catalytic subunit & 2.2046 & 3.9274 \\
\hline \multirow{9}{*}{$\mathrm{C}$} & gi|77628000 & Q497B0 & NIT2 & Nit2 & omega-amidase NIT2 & 1.5374 & 1.1864 \\
\hline & gi|13928690 & P41562 & IDHC & $I d h 1$ & isocitrate dehydrogenase [NADP] cytoplasmic & 1.5688 & 1.3028 \\
\hline & gi|220684 & $\mathrm{P} 13221$ & AATC & Got1 & cytosolic aspartate aminotransferase & 1.5696 & 1.0949 \\
\hline & gi|110625641 & Q7TP54 & Q7TP54 & Fam65b, RGD1306939 & family with sequence similarity 65 , member B & 1.6062 & 1.4191 \\
\hline & gi|17865351 & P46462 & TERA & $V c p$ & transitional endoplasmic reticulum ATPase & 1.7281 & 1.2094 \\
\hline & gi|71896574 & Q9WUW8 & ST1C2 & Sult1c2 Sultk1 & sulfotransferase $1 \mathrm{C} 2$ & 1.8846 & 1.7668 \\
\hline & gi|157822207 & D4A2K1 & D4A2K1 & $\begin{array}{l}\text { Hogal, } R G D 1310475 \\
\text { predicted rCG_57749 }\end{array}$ & dihydrodipicolinate synthase-like, mitochondrial & 1.9459 & 1.1041 \\
\hline & gi|155369281 & A2VCW9 & AASS & Aass & $\begin{array}{l}\text { alpha-aminoadipic semialdehyde synthase, mitochondri- } \\
\text { al precursor }\end{array}$ & 2.4768 & 1.6490 \\
\hline & gi|1657632 & P70619 & GSHR & Gsr & glutathione reductase & 2.8381 & 1.4945 \\
\hline
\end{tabular}



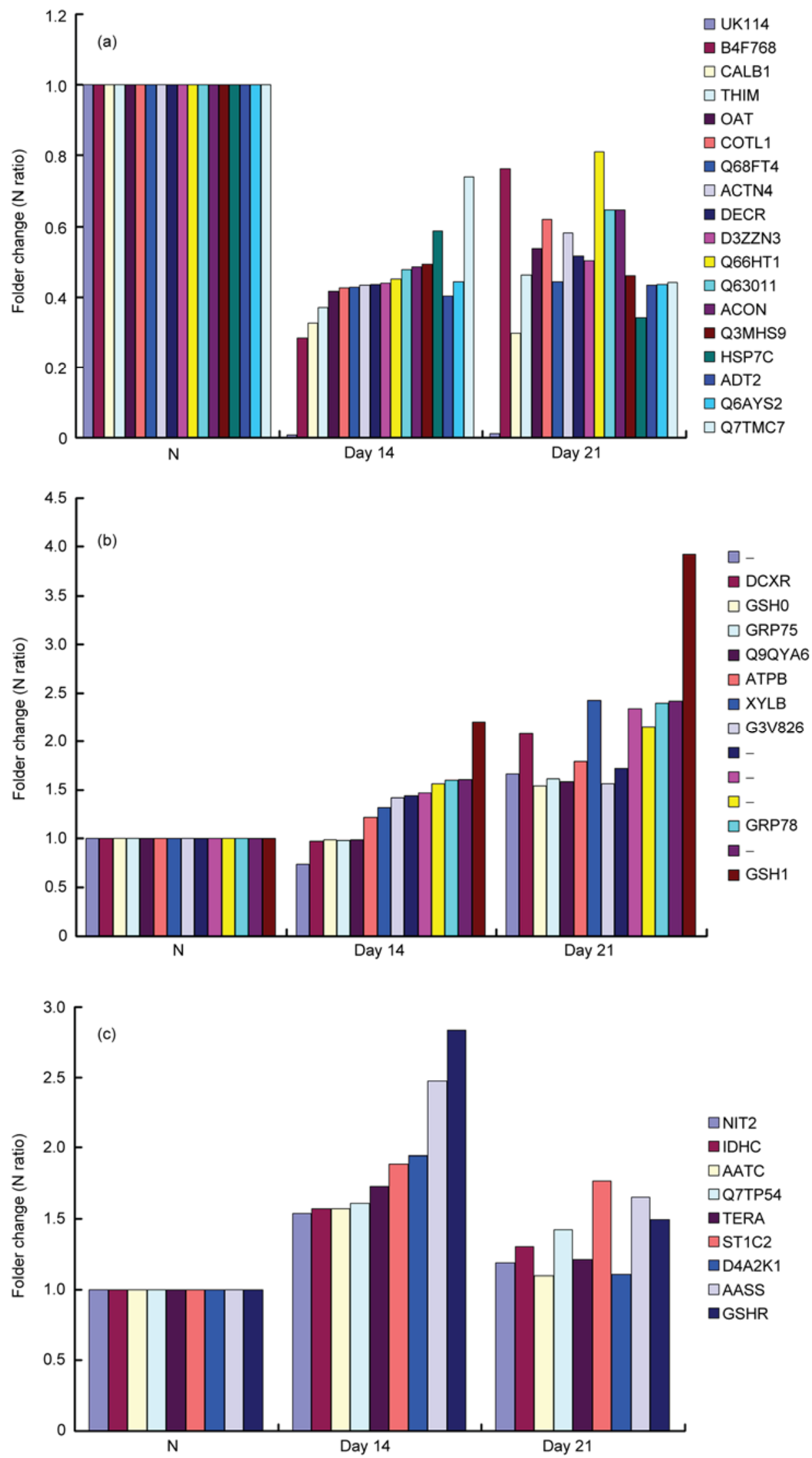

Figure 1 All differentially expressed proteins were divided into three categories. (a) The expression of proteins was downregulated at day 14, but upregulated at day 21. (b) The expression of proteins was downregulated at days 14 and 21. (c) The expression of proteins was upregulated at days 14 and 21. 
(a)

(b)

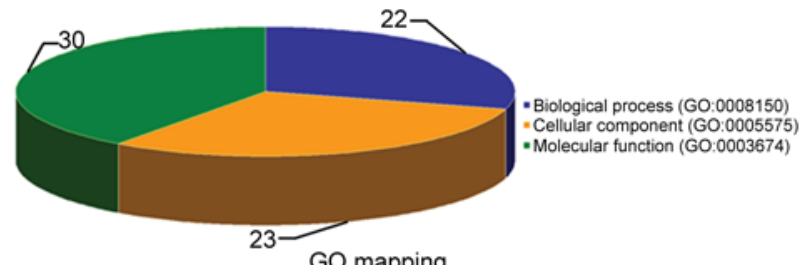

GO mapping

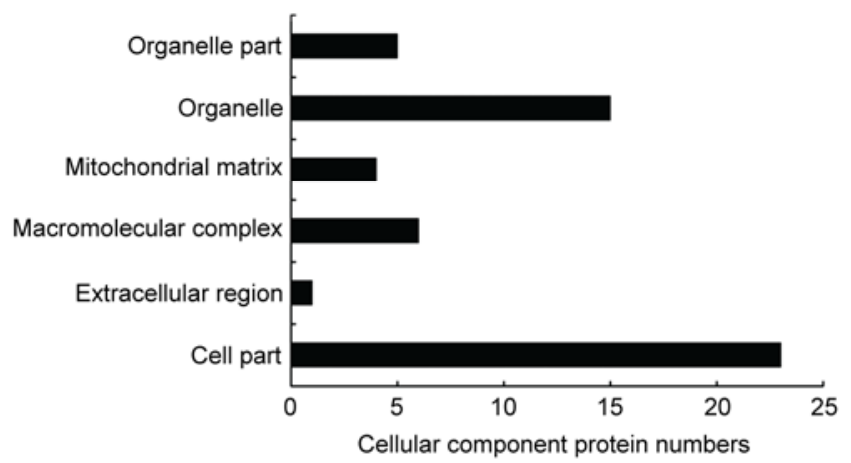

(c)

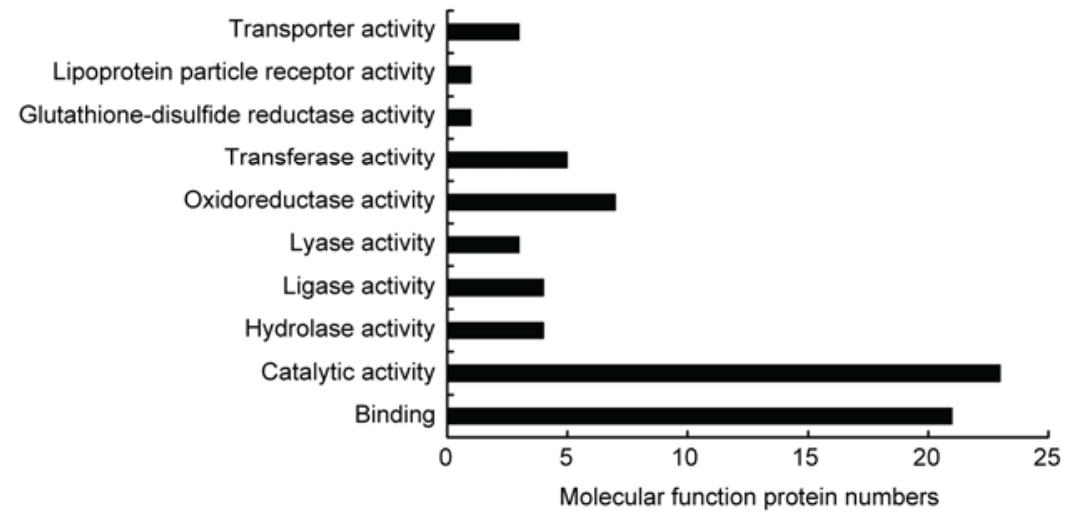

(d)

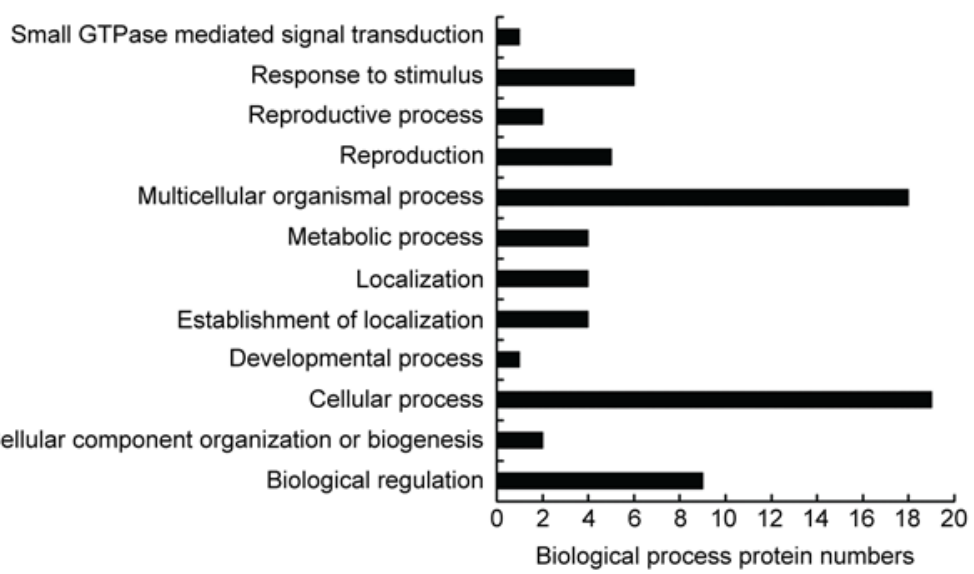

Figure 2 GO analyses of all differentially expressed proteins. (a) There are 23 proteins involved in cellular components, 30 proteins in molecular function, and 22 proteins in biological processes. (b) Protein numbers in the subgroup of cellular components. (c) Protein numbers in the subgroup of molecular function. (d) Protein numbers in biological processes.

\subsection{Analyses of VCP protein}

Protein-GO networks of all proteins are provided in Figure $\mathrm{S} 1$. Combining these results, we analyzed the interaction of proteins using an MAS (Molecular Annotation System). VCP (fold change at day 14 was 1.7281 , and 1.2094 at day
21 ) interacted with many other proteins, suggesting that it plays an important role in the renal disease process. The VCP-GO networks are shown in Figure 3. The results indicated that HSPa5, markers of ERS, could interact with VCP via slac2a4 (omitted in Table 1). We also extracted the detailed GO function of VCP in Table 4, which showed that 
Table 2 Cellular metabolic process related proteins

\begin{tabular}{|c|c|c|}
\hline Uniprot AC & Protein name & Gene Ontology (GO) \\
\hline P13221 & AATC & $\begin{array}{l}\text { 2-oxoglutarate metabolic process; } L \text {-phenylalanine:2-oxoglutarate aminotransferase activity; axon terminus; biosynthetic } \\
\text { process; carboxylic acid binding; cytosol; glutamate metabolic process; lysosome }\end{array}$ \\
\hline Q9ER34 & ACON & $\begin{array}{l}3 \text { iron, } 4 \text { sulfur cluster binding; } 4 \text { iron, } 4 \text { sulfur cluster binding; aconitate hydratase activity; citrate hydro-lyase (cis-aconitate- } \\
\text { forming) activity; citrate metabolic process; isocitrate hydro-lyase (cis-aconitate-forming) activity; isocitrate metabolic process }\end{array}$ \\
\hline P10719 & АТРВ & $\begin{array}{l}\text { ADP biosynthetic process; ATP binding; ATP hydrolysis coupled proton transport; ATP synthesis coupled proton transport; } \\
\text { calcium ion binding; rotational mechanism; lipoprotein particle receptor activity; mitochondrial proton-transporting ATP } \\
\text { synthase complex }\end{array}$ \\
\hline Q920P0 & DCXR & $\begin{array}{l}D \text {-xylose metabolic process; } L \text {-xylulose reductase }\left(\mathrm{NADP}^{+}\right) \text {activity; glucose metabolic process; membrane; nucleotide bind- } \\
\text { ing; protein homotetramerization; xylulose metabolic process }\end{array}$ \\
\hline P48508 & GSHO & $\begin{array}{l}\text { glutamate metabolic process; glutamate-cysteine ligase activity; glutamate-cysteine ligase complex; glutathione biosynthetic } \\
\text { process; negative regulation of neuron apoptosis; response to nitrosative stress; response to oxidative stress; soluble fraction }\end{array}$ \\
\hline P19468 & GSH1 & $\begin{array}{l}\text { ADP binding; anti-apoptosis; cell redox homeostasis; coenzyme binding; cysteine metabolic process; glutamate binding; } \\
\text { glutamate metabolic process; glutathione biosynthetic process; response to heat; response to hormone stimulus; response to } \\
\text { oxidative stress }\end{array}$ \\
\hline P70619 & GSHR & $\begin{array}{l}\text { NADP binding; cell redox homeostasis; cytosol; flavin adenine dinucleotide binding; glutathione binding; glutathione meta- } \\
\text { bolic process; glutathione-disulfide reductase activity; mitochondrion; protein homodimerization activity; soluble fraction; } \\
\text { spermatogenesis }\end{array}$ \\
\hline P41562 & IDHC & $\begin{array}{l}\text { 2-oxoglutarate metabolic process; NAD binding; NADP binding; female gonad development; glyoxylate cycle; isocitrate } \\
\text { dehydrogenase }\left(\mathrm{NADP}^{+}\right) \text {activity; isocitrate metabolic process; magnesium ion binding; peroxisome; response to organic } \\
\text { cyclic compound }\end{array}$ \\
\hline P04182 & OAT & $\begin{array}{l}\text { N2-acetyl- } L \text {-ornithine:2-oxoglutarate } 5 \text {-aminotransferase activity; mitochondrial matrix; ornithine metabolic process; orni- } \\
\text { thine-oxo-acid transaminase activity; pyridoxal phosphate binding }\end{array}$ \\
\hline P13437 & THIM & $\begin{array}{l}\text { acetyl-CoA C-acyltransferase activity; acetyl-CoA metabolic process; anti-apoptosis; fatty acid beta-oxidation; mitochondrial } \\
\text { matrix }\end{array}$ \\
\hline Q3MIF4 & XYLB & ATP binding; $D$-xylose metabolic process; xylulokinase activity \\
\hline Q3MHS9 & Q3MHS9 & ATP binding; cytoplasm; protein folding; unfolded protein binding \\
\hline D3ZZN3 & D3ZZN3 & AMP binding; acetate biosynthetic process; acetate-CoA ligase activity; propionate biosynthetic process \\
\hline Q66HT1 & Q66HT1 & fructose-bisphosphate aldolase activity; glycolysis \\
\hline B4F768 & B4F768 & $\begin{array}{l}\text { 1-pyrroline-5-carboxylate dehydrogenase activity; mitochondrial matrix; oxidoreductase activity, acting on the aldehyde or } \\
\text { oxo group of donors, NAD or NADP as acceptor; proline biosynthetic process }\end{array}$ \\
\hline Q68FT4 & Q68FT4 & $\begin{array}{l}\text { ATP binding; succinate metabolic process; succinate-CoA ligase activity; succinyl-CoA metabolic process; tricarboxylic acid } \\
\text { cycle }\end{array}$ \\
\hline
\end{tabular}

it likely has 14 functions, including ER to Golgi vesiclemediated transport, proteasomal ubiquitin-dependent protein catabolism, and protein homooligomerization (Figure 3).

\section{5 mRNA and protein expression of VCP and HSPa5 in the model}

In the LC-MS/MS results, the expression of the two proteins at days 14 and 21 is shown in Figure 4(a), which includes groups $\mathrm{B}$ and $\mathrm{C}$. To verify expression of the two proteins in the three groups, we detected the mRNA and protein expression level using real-time PCR and Western blotting. The results (Figure 4(b) and (c)) indicated that at days 14 and 21 , the mRNA and protein expression level of VCP and HSPa5 were upregulated when compared to normal tissue. Although at day 21, the expression of VCP was downregulated, the difference between the two time points was not statistically significant.

\subsection{Pathway analysis}

All significant pathways in which these differentially expressed proteins were involved are shown in Figure S1 and Table 5. The main pathways include glutathione metabolism, reductive carboxylate cycle $\left(\mathrm{CO}_{2}\right.$ fixation), citrate cycle, arginine and proline metabolism, and control of genes including Gclm, Gclc, Idh1, Gsr, Acss1, Aco2, Idh1, Suclg2, Aldh4al, Oat, and Gotl. We obtained the correlation of these pathway related proteins/genes, as shown in Figure 4. VCP and HSPa5 were involved in the same pathways.

\subsection{ERS of podocyte after inhibiting VCP}

After treatment of MPCs with tunicamycin for $10 \mathrm{~h}$, the expression level of HSPa5, the ERS marker, was upregulated, as shown in Figure 5. Expression of VCP also increased. If cell VCP was inhibited by siRNA, HSPa5 expression did not change. After the cell RNAi was treated 
Table 3 Catalytic activity related proteins

\begin{tabular}{|c|c|c|}
\hline Uniprot AC & Protein name & Gene Ontology (GO) \\
\hline A2VCW9 & AASS & $\begin{array}{l}\text { mitochondrion; nucleotide binding; saccharopine dehydrogenase ( } \mathrm{NAD}^{+}, L \text {-glutamate-forming) activity; saccharopine dehy- } \\
\text { drogenase (NADP }{ }^{+}, L \text {-lysine-forming) activity }\end{array}$ \\
\hline P13221 & AATC & 2-oxoglutarate metabolic process; cytosol; glutamate metabolic process; lysosome; pyridoxal phosphate binding; soluble fraction \\
\hline Q9ER34 & ACON & $\begin{array}{l}3 \text { iron, } 4 \text { sulfur cluster binding; } 4 \text { iron, } 4 \text { sulfur cluster binding; isocitrate metabolic process; mitochondrion; protein binding; } \\
\text { tricarboxylic acid cycle }\end{array}$ \\
\hline P10719 & ATPB & $\begin{array}{l}\text { ADP biosynthetic process; ATP binding; ATP hydrolysis coupled proton transport; mitochondrial proton-transporting ATP } \\
\text { synthase complex, catalytic core F(1) }\end{array}$ \\
\hline Q920P0 & DCXR & $\begin{array}{l}D \text {-xylose metabolic process; } L \text {-xylulose reductase }\left(\mathrm{NADP}^{+}\right) \text {activity; glucose metabolic process; membrane; nucleotide bind- } \\
\text { ing; protein homotetramerization; xylulose metabolic process }\end{array}$ \\
\hline Q64591 & DECR & 2,4-dienoyl-CoA reductase (NADPH) activity; mitochondrion \\
\hline P48508 & GSHO & $\begin{array}{l}\text { glutamate metabolic process; glutamate-cysteine ligase activity; glutamate-cysteine ligase catalytic subunit binding; gluta- } \\
\text { mate-cysteine ligase complex; glutathione biosynthetic process; negative regulation of neuron apoptosis; response to oxidative } \\
\text { stress; soluble fraction }\end{array}$ \\
\hline P19468 & GSH1 & $\begin{array}{l}\text { ADP binding; anti-apoptosis; cell redox homeostasis; coenzyme binding; cysteine metabolic process; glutamate binding; glu- } \\
\text { tamate-cysteine ligase complex; glutathione biosynthetic process; magnesium ion binding; negative regulation of neuron apop- } \\
\text { tosis; response to oxidative stress }\end{array}$ \\
\hline P70619 & GSHR & $\begin{array}{l}\text { NADP binding; cell redox homeostasis; cytosol; flavin adenine dinucleotide binding; glutathione binding; glutathione meta- } \\
\text { bolic process; glutathione-disulfide reductase activity; mitochondrion; protein homodimerization activity; soluble fraction; } \\
\text { spermatogenesis }\end{array}$ \\
\hline P41562 & IDHC & $\begin{array}{l}\text { 2-oxoglutarate metabolic process; NAD binding; peroxisome; response to organic cyclic compound; response to steroid hor- } \\
\text { mone stimulus; soluble fraction; tricarboxylic acid cycle }\end{array}$ \\
\hline Q497B0 & NIT2 & nitrogen compound metabolic process; omega-amidase activity \\
\hline P04182 & OAT & $\begin{array}{l}\text { N2-acetyl- } L \text {-ornithine:2-oxoglutarate } 5 \text {-aminotransferase activity; mitochondrial matrix; ornithine metabolic process; orni- } \\
\text { thine-oxo-acid transaminase activity; pyridoxal phosphate binding }\end{array}$ \\
\hline Q9WUW8 & ST1C2 & lysosome; sulfotransferase activity \\
\hline P46462 & TERA & $\begin{array}{l}\text { ATP binding; ATPase activity; ER to Golgi vesicle-mediated transport; cytosol; endoplasmic reticulum; identical protein bind- } \\
\text { ing; lipid binding; microsome; nucleus; positive regulation of proteasomal ubiquitin-dependent protein catabolic process; pro- } \\
\text { tein homooligomerization; receptor binding }\end{array}$ \\
\hline P13437 & THIM & $\begin{array}{l}\text { acetyl-CoA C-acyltransferase activity; acetyl-CoA metabolic process; anti-apoptosis; fatty acid beta-oxidation; mitochondrial } \\
\text { matrix }\end{array}$ \\
\hline P52759 & UK114 & endonuclease activity; mitochondrion; nucleus; peroxisome \\
\hline Q3MIF4 & XYLB & ATP binding; $D$-xylose metabolic process; xylulokinase activity \\
\hline D4A2K1 & D4A2K1 & lyase activity \\
\hline Q7TP54 & Q7TP54 & cellular iron ion homeostasis; ferric iron binding; oxidoreductase activity \\
\hline D3ZZN3 & D3ZZN3 & AMP binding; acetate biosynthetic process; acetate-CoA ligase activity; propionate biosynthetic process \\
\hline Q66HT1 & Q66HT1 & fructose-bisphosphate aldolase activity; glycolysis \\
\hline B4F768 & B4F768 & $\begin{array}{l}\text { 1-pyrroline-5-carboxylate dehydrogenase activity; mitochondrial matrix; oxidoreductase activity, acting on the aldehyde or oxo } \\
\text { group of donors, NAD or NADP as acceptor; proline biosynthetic process }\end{array}$ \\
\hline
\end{tabular}

with tunicamycin, the VCP expression level was lower than that of the group treated with only tunicamycin, but the HSPa5 expression level was significantly higher.

\section{Discussion}

Membranous glomerulonephritis (MGN) accounts for approximately $30 \%$ of cases of nephrotic syndrome in adults, with peak incidence between the ages of 30 and 50 years and a male to female ratio of $2: 1$ [19]. Major contributions to our current understanding of the disease come from
Heymann nephritis, a rat model of MGN [20]. The pathological features of membranous nephropathy are uniform and consistent incrassation of the glomerular capillary basement membrane, and diffuse subepithelial immune complex deposition. An obvious increase in proteinuria was observed in the pHN by day 14 and it peaked on day 21; on this day, interstitial fibrosis appeared [21]. Therefore, we selected days 14 and 21 as the two time points of the pHN model to analyze the differential protein expression profile using label-free LC-MS/MS. Proteomics applied at a large scale may provide useful diagnostic information. Several reports on $\mathrm{MN}$ have focused on the urine for diagnostic purposes 
(a)

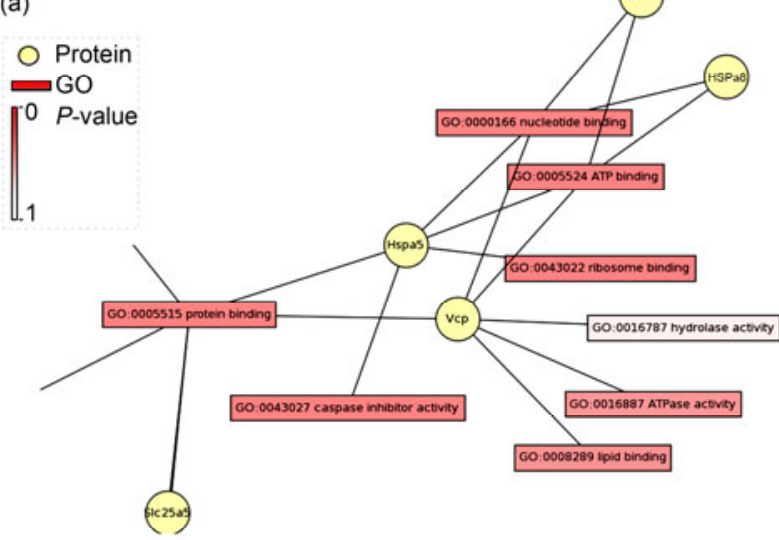

(c)

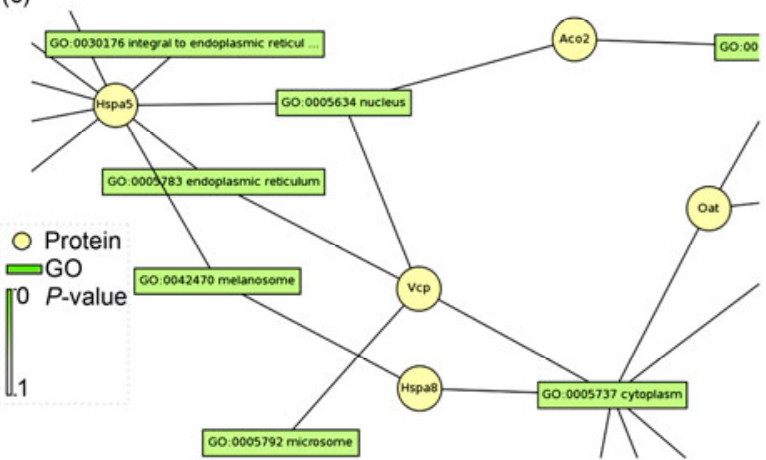

(b)
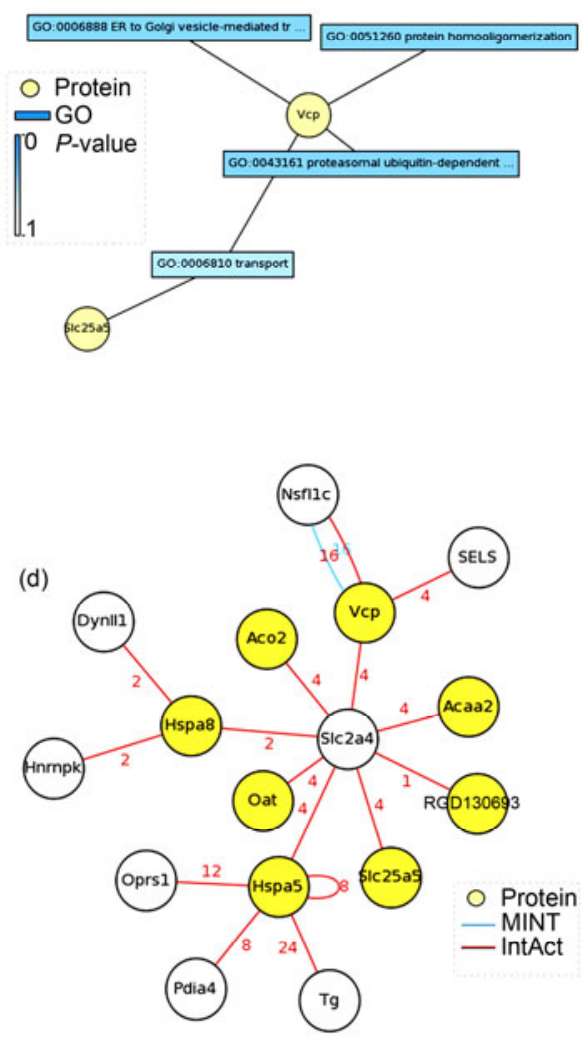

Figure 3 Protein-GO networks and interaction with VCP. (a) Significant molecular function network of VCP. (b) Significant biological process network of VCP. (c) Significant cellular component network of VCP. (d) Interaction graph of VCP; the numbers represent the frequency of interaction.
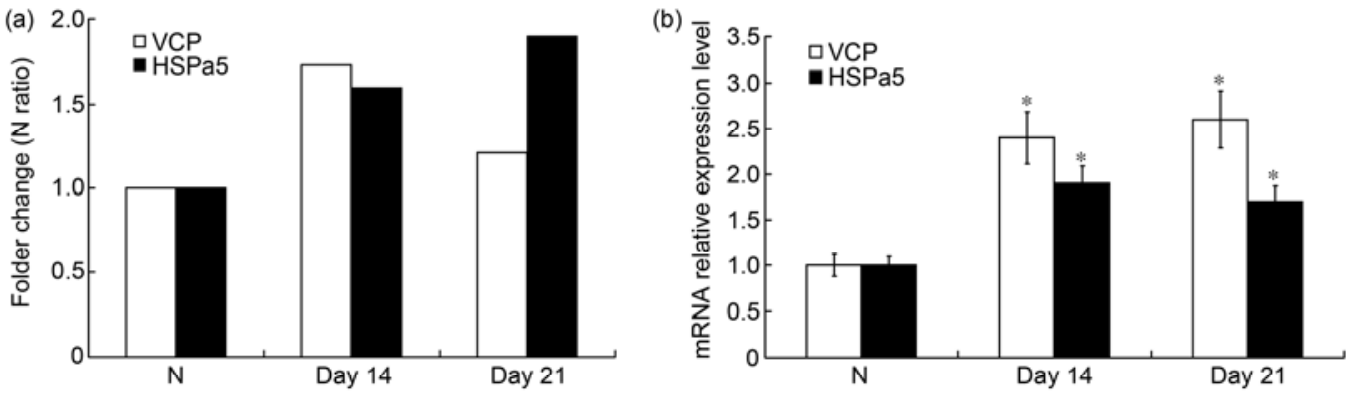

(c)

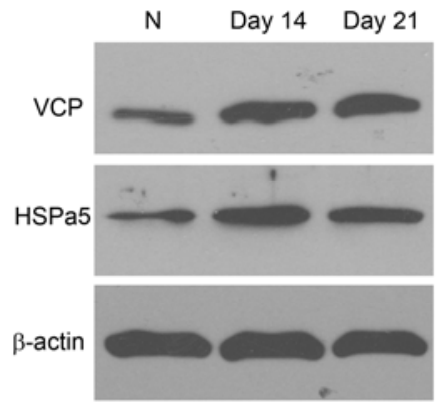

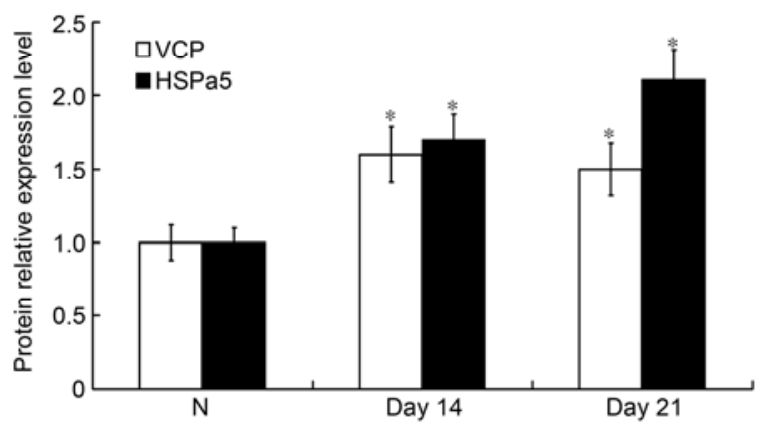

Figure 4 Verification of VCP and HSPa5 in LC-MS/MS by real-time PCR and Western blotting. (a) The degree of change of VCP and HSPa5 on days 14 and 21 in the pHN model detected by LC-MS/MS. (b) Real-time PCR determined the mRNA expression level of VCP and HSPa5; at days 14 and 21, the two proteins showed increased expression, and significant differences were observed between days 14 and 21 compared to normal tissue. $* P<0.05, n=3$. (c) Western blotting determined the protein expression level of VCP and HSPa5; at days 14 and 21, the two proteins showed increased expression, and significant differences were observed between days 14 and 21 compared to normal tissue. $* P<0.05, n=3$. 
Table 4 The detailed GO analysis of VCP protein (GI:1786535)

\begin{tabular}{lll}
\hline \multicolumn{1}{c}{ Molecular function } & \multicolumn{1}{c}{ Biological process } & Cellular component \\
\hline GO:0000166 nucleotide binding & GO:0006810 transport & GO:0005634 nucleus \\
GO:0005515 protein binding & GO:0006888 ER to Golgi vesicle-mediated transport & GO:0005737 cytoplasm \\
GO:0005524 ATP binding & GO:0043161 proteasomal ubiquitin-dependent protein catabolism & GO:0005783 \\
GO:0008289 lipid binding & GO:0051260 protein homooligomerization & GO:0005792 \\
GO:0016787 hydrolase activity & & microsome \\
GO:0016887 ATPase activity & & \\
\hline
\end{tabular}

Table 5 Pathway information of differentially expressed proteins ${ }^{\text {a) }}$

\begin{tabular}{|c|c|c|c|c|c|}
\hline Pathway & Count & $P$-value & $Q$-value & Gene & GI number \\
\hline Glutathione metabolism & 4 & $3.36 \times 10^{-8}$ & $1.68 \times 10^{-8}$ & Gclm; Gclc; Idh $;$; Gsr & $8393446 ; 25742748 ; 13928690 ; 1657632$ \\
\hline Reductive carboxylate cycle $\left(\mathrm{CO}_{2}\right.$ fixation $)$ & 3 & $5.21 \times 10^{-8}$ & $2.08 \times 10^{-8}$ & Acss $1 ;$ Aco $2 ; \operatorname{Idh} 1$ & $157818027 ; 40538860 ; 13928690$ \\
\hline Citrate cycle (TCA cycle) & 3 & $1.74 \times 10^{-6}$ & $5.81 \times 10^{-7}$ & Suclg2; Aco2; Idh1 & $51260799 ; 40538860 ; 13928690$ \\
\hline Arginine and proline metabolism & 3 & $2.13 \times 10^{-6}$ & $6.08 \times 10^{-7}$ & Aldh4a1; Oat; Got 1 & $195540087 ; 11968102 ; 220684$ \\
\hline Pentose phosphate pathway & 2 & $1.63 \times 10^{-4}$ & $2.52 \times 10^{-5}$ & Aldob; Tkt; Aldob & $158081751 ; 149034221 ; 149020188$ \\
\hline Glutamate metabolism & 2 & $1.77 \times 10^{-4}$ & $2.52 \times 10^{-5}$ & Aldh4al; Got1 & $195540087 ; 220684$ \\
\hline Alanine and aspartate metabolism & 2 & $2.22 \times 10^{-4}$ & $2.62 \times 10^{-5}$ & Agxt $2 ;$ Got 1 & $149027328 ; 220684$ \\
\hline Propanoate metabolism & 2 & $2.73 \times 10^{-4}$ & $3.03 \times 10^{-5}$ & Suclg2; Acss 1 & $51260799 ; 157818027$ \\
\hline Glycolysis/Gluconeogenesis & 2 & 0.002702 & $2.46 \times 10^{-4}$ & Acss $1 ;$ Aldob; Aldob & $157818027 ; 158081751 ; 149020188$ \\
\hline Antigen processing and presentation & 2 & 0.002866 & $2.49 \times 10^{-4}$ & Hspa $8 ;$ Hspa 5 & $13242237 ; 25742763$ \\
\hline Alkaloid biosynthesis I & 1 & 0.004703 & $3.62 \times 10^{-4}$ & Got1 & 220684 \\
\hline Fatty acid elongation in mitochondria & 1 & 0.007827 & $4.89 \times 10^{-4}$ & Acaa2 & 18426866 \\
\hline $\begin{array}{l}\text { Phenylalanine, tyrosine and tryptophan } \\
\text { biosynthesis }\end{array}$ & 1 & 0.007827 & $4.89 \times 10^{-4}$ & Got1 & 220684 \\
\hline Glyoxylate and dicarboxylate metabolism & 1 & 0.008607 & $4.92 \times 10^{-4}$ & Aco2 & 40538860 \\
\hline Parkinson's disease & 2 & 0.008613 & $4.92 \times 10^{-4}$ & Slc25a5; Atp $5 b$ & $32189350 ; 203033$ \\
\hline Cysteine metabolism & 1 & 0.010164 & $5.56 \times 10^{-4}$ & Got1 & 220684 \\
\hline Phenylalanine metabolism & 1 & 0.010942 & $5.61 \times 10^{-4}$ & Got1 & 220684 \\
\hline Pentose and glucuronate interconversions & 1 & 0.017142 & $7.79 \times 10^{-4}$ & $X y l b$ & 76096348 \\
\hline Bile acid biosynthesis & 1 & 0.018686 & $8.21 \times 10^{-4}$ & Acaa2 & 18426866 \\
\hline Tyrosine metabolism & 1 & 0.023305 & $9.51 \times 10^{-4}$ & Got1 & 220684 \\
\hline Fructose and mannose metabolism & 1 & 0.024839 & $9.94 \times 10^{-4}$ & Aldob; Aldob & $158081751 ; 149020188$ \\
\hline Pyruvate metabolism & 1 & 0.028666 & 0.001102553 & Acss 1 & 157818027 \\
\hline Fatty acid metabolism & 1 & 0.031718 & 0.001185701 & Acaa2 & 18426866 \\
\hline Glycine, serine and threonine metabolism & 1 & 0.031718 & 0.001185701 & Agxt 2 & 149027328 \\
\hline Valine, leucine and isoleucine degradation & 1 & 0.034759 & 0.001241406 & Acaa2 & 18426866 \\
\hline Adherens junction & 1 & 0.055052 & 0.001931646 & Actn 4 & 6636119 \\
\hline Leukocyte transendothelial migration & 1 & 0.087242 & 0.002814248 & Actn 4 & 6636119 \\
\hline Tight junction & 1 & 0.095122 & 0.002860587 & Actn4 & 6636119 \\
\hline Systemic lupus erythematosus & 1 & 0.105057 & 0.002959349 & Actn 4 & 6636119 \\
\hline Oxidative phosphorylation & 1 & 0.117675 & 0.003159073 & Atp $5 b$ & 203033 \\
\hline Focal adhesion & 1 & 0.137645 & 0.003536344 & Actn4 & 6636119 \\
\hline Calcium signaling pathway & 1 & 0.139006 & 0.003536344 & Slc25a5 & 32189350 \\
\hline Regulation of actin cytoskeleton & 1 & 0.149147 & 0.003699245 & Actn4 & 6636119 \\
\hline Alzheimer's disease & 1 & 0.178885 & 0.004160114 & Atp $5 b$ & 203033 \\
\hline MAPK signaling pathway & 1 & 0.185356 & 0.004179774 & Hspa8 & 13242237 \\
\hline
\end{tabular}

a) $P$-value: we calculate the $P$ value using a hypergeometric distribution. The genes are enriched significantly in the pathway when $P$ value $<0.05 . Q$ value: $Q$ is Fasle Discovery Rate (FDR), $Q$ default 0.05 , the less the $Q$ is, the more significant the genes (or proteins) enriched in the one pathway (or GO term), and the less FDR. 

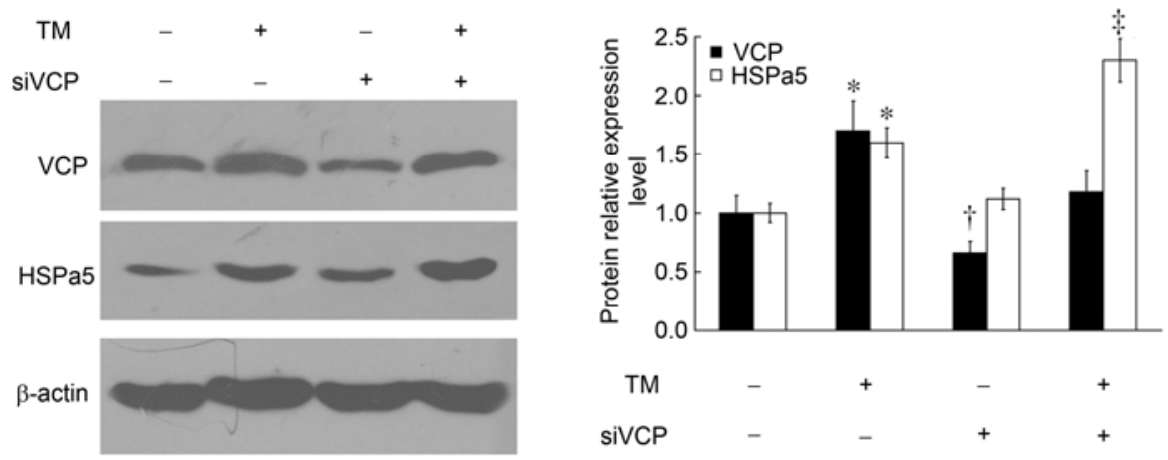

Figure 5 VCP was inhibited by RNAi, and ERS was induced by tunicamycin (Tm). MPCs were pretreated with $10 \mu \mathrm{g} / \mathrm{mL}$ Tm for $10 \mathrm{~h}$ to induce ERS; the expression levels of VCP and HSPa5 were both upregulated, and significant differences were observed compared with the control. $* P<0.05, n=3$. MPCs were transfected with siRNA and VCP protein expression was inhibited ( $\uparrow P<0.05, n=3$ ), but HSPa5 expression did not change. Cells were transfected with siRNA of VCP and treated with Tm; HSPa5 expression increased, and a significant difference was observed between the Tm and Tm+siRNA groups $(\$ P<$ $0.05, n=3$ ), although VCP expression was considerable in the control.

$[22,23]$. However, few reports have analyzed the differentially expressed proteins in the development of MN. Our objective was to identify the differentially expressed proteins seen in the pHN rat model. The results identified 41 differentially expressed proteins among three groups (day 0 , day 14, and day 21). According to their expression changes, we divided them into three categories, A, B, and C. Proteins in group A were downregulated at day 14, but upregulated at day 21; group B showed downregulation at days 14 and 21; and group $\mathrm{C}$ showed upregulation at days 14 and 21 . These proteins played an important role in the cellular process and biological regulation (listed in Table 1). The 16 proteins related to cellular metabolism were the predominant components. Since ERS profoundly affects metabolic processes [24], our findings strongly suggest that ERS on these proteins might be one of the most important biological events at day 14 or 21 in the PHN rat model. In the ERS process, misfolded protein in the ER would be degraded in the cytoplasm by catalytic proteins [25-27]. Table 3 lists the catalytic proteins (22) and their GO terms. These proteins affect the ERS process.

$\mathrm{HSPa} 8$ and HSPa5, the proteins needed for cellular metabolism, belong to the heat-shock protein family, and can bind to nascent polypeptides to facilitate correct folding $[28,29]$. They also function as an ATPase in the disassembly of clathrin-coated vesicles during transport of membrane components through the cell $[30,31]$. VCP, the catalytic protein, belongs to a family that includes putative ATP-binding proteins involved in vesicle transport and fusion, 26S proteasome function, and assembly of peroxisomes [32]. It also binds with clathrin and heat-shock protein Hsc70 to form a complex [33]. Additionally, it has been implicated in several other cellular events related to mitosis, including homotypic membrane fusion and ubiquitin-dependent protein degradation [34-36]. Our results indicate that most differentially expressed proteins could interact with each other, as shown in Figure 2, and among these, VCP could interact with HSPa8 or HSPa5 via slc2a4. Re- grettably, our LC-MS/MS did not provide evidence of a significant change in slc24a expression. Our findings suggest that the cell processes mediated by VCP affect the development of MN.

Research indicates that the basic mechanism of $\mathrm{MN}$ is complement-mediated injury of the podocyte and its slitpore membrane $[37,38]$ and that ERS is the main cause of podocyte injury $[39,40]$. HSPa8 and HSPa5 are considered the markers of ERS increase [41-43], and VCP is an integral component of the ER-associated degradation and cellular stress pathways $[44,45]$. The detailed GO analysis of VCP in our study agreed with this finding (Table 4), and the pathway analyses showed VCP and HSPa5 to be in the same functional pathway. Therefore, we hypothesized that ERS, with VCP as a mediator, is an important trigger of podocyte injury in $\mathrm{MN}$.

To test this theory, we cultured MPCs and induced ERS with tunicamycin, knocking down the expression level by RNAi. Results suggested when VCP of cells was knocked down under normal conditions, the ERS did not change, but VCP knockdown could increase ERS significantly after treatment with tunicamycin. Gp78-VCP interaction may represent one means of degradation of ER-associated degradation substrates [46]. VCP is required for maintenance of normal ER structure and function and mediates the degradation of certain proteins [47]. This supports our results that under normal conditions, the podocyte ERS is unaffected by VCP, but under stress, VCP may be activated to degrade unfolded proteins.

In conclusion, ERS plays an important role in podocyte injury of MN, and it is mediated via the HSPa5-VCP signaling pathway. In this process, the cellular metabolism proteins and catalytic proteins were predominant.

This work was supported by the National Natural Science Foundation of China (81102673 and 31170810) and the National Basic Research Program of China (2007CB507400). We thank Ms. Liaoshao (Cloud Scientific Technology Co. Ltd. China) for her support in the data analysis. 
1 Sharpstone P, Ogg C S, Cameron J S. Nephrotic syndrome due to primary renal disease in adults: I. Survey of incidence in south east England. Br Med J, 1969, 2: 533-535

2 Rivera F, Lopez-Gomez J M, Perez-Garcia R. Clinicopathologic correlations of renal pathology in Spain. Kidney Int, 2004, 66: 898-904

3 Chen Z H, Qin W S, Zeng C H, et al. Triptolide reduces proteinuria in experimental membranous nephropathy and protects against $\mathrm{C} 5 \mathrm{~b}$ 9-induced podocyte injury in vitro. Kidney Int, 2010, 77: 974-988

4 Nangaku M, Pippin J, Richardson C A, et al. Beneficial effects of systemic immunoglobulin in experimental membranous nephropathy. Kidney Int, 1996, 50: 2054-2062

5 Jefferson J A, Pippin J W, Shankland S J. Experimental models of membranous nephropathy. Drug Discov Today Dis Models, 2010, 7: 27-33

6 Wu Q, Fan K, Sha W, et al. Highly sensitive detection of melamine based on reversed phase liquid chromatography mass spectrometry. Chin Sci Bull, 2009, 54: 732-737

7 Old W M, Meyer-Arendt K, Aveline-Wolf L, et al. Comparison of label-free methods for quantifying human proteins by shotgun proteomics. Mol Cell Proteomics, 2005, 4: 1487-1502

8 Asara J M, Christofk H R, Freimark L M, et al. A label-free quantification method by MS/MS TIC compared to SILAC and spectral counting in a proteomics screen. Proteomics, 2008, 8: 994-999

9 Dayarathna M K, Hancock W S, Hincapie M. A two step fractionation approach for plasma proteomics using immunodepletion of abundant proteins and multi-lectin affinity chromatography: Application to the analysis of obesity, diabetes, and hypertension diseases. $\mathbf{J}$ Sep Sci, 2008, 31: 1156-1166

10 Patel V J, Thalassinos K, Slade S E, et al. A comparison of labeling and label-free mass spectrometry-based proteomics approaches. J Proteome Res, 2009, 8: 3752-3759

11 Ke C Y, Geng X D. A new chromatographic method for fast separation of active proteins. Chin Sci Bull, 2008, 53: 1113-1116

12 Chant S, Katz A, Silverman M. Pathogenicity of a highly purified brush border membrane preparation in Heymann nephritis. J Clin Lab Immunol, 1980, 4: 133-140

13 Noble B, Van Liew J B, Andres G A, et al. Factors influencing susceptibility of LEW rats to Heymann nephritis. Clin Immunol Immunopathol, 1984, 30: 241-254

14 Bhan A K, Schneeberger E E, Baird L G, et al. Studies with monoclonal antibodies against brush border antigens in Heymann nephritis. Lab Invest, 1985, 53: 421-432

15 Shankland S J, Pippin J W, Reiser J, et al. Podocytes in culture: Past, present, and future. Kidney Int, 2007, 72: 26-36

16 Quan H, Peng X, Yang L, et al. An in-depth analysis of proteomics expression profiling in rat glomeruli utilizing LC-MS. Chin Sci Bull, 2010, 55: 2142-2151

17 Apweiler R, Bairoch A, Wu C H, et al. UniProt: The Universal Protein knowledgebase. Nucleic Acids Res, 2004, 32: D115-119

18 Yuan J S, Reed A, Chen F, et al. Statistical analysis of real-time PCR data. BMC Bioinformatics, 2006, 7: 85

19 Fauci A S, Braunwald E, Kasper D L, et al. Harrison's Principles of Internal Medicine. 17th ed. New York: McGraw-Hill, 2008. 1789-1790

20 Heymann W, Hackel D B, Harwood S, et al. Production of nephrotic syndrome in rats by Freund's adjuvants and rat kidney suspensions. Proc Soc Exp Biol Med, 1959, 100: 660-664

21 Bonegio R G, Fuhro R, Wang Z, et al. Rapamycin ameliorates proteinuria-associated tubulointerstitial inflammation and fibrosis in experimental membranous nephropathy. J Am Soc Nephrol, 2005, 16: 2063-2072

22 Weissinger E M, Wittke S, Kaiser T, et al. Proteomic patterns established with capillary electrophoresis and mass spectrometry for diagnostic purposes. Kidney Int, 2004, 65: 2426-2434

23 Ngai H H, Sit W H, Jiang P P, et al. Serial changes in urinary proteome profile of membranous nephropathy: Implications for pathophysiology and biomarker discovery. J Proteome Res, 2006, 5: 30383047

24 Wang X, Eno C O, Altman B J, et al. ER stress modulates cellular metabolism. Biochem J, 2011, 435: 285-296
25 Johnston J A, Ward C L, Kopito R R. Aggresomes: A cellular response to misfolded proteins. J Cell Biol, 1998, 143: 1883-1898

26 Katiyar S, Li G, Lennarz W J. A complex between peptide: $\mathrm{N}$-glycanase and two proteasome-linked proteins suggests a mechanism for the degradation of misfolded glycoproteins. Proc Natl Acad Sci USA, 2004, 101: 13774-13779

27 Lilley B N, Ploegh H L. Multiprotein complexes that link dislocation, ubiquitination, and extraction of misfolded proteins from the endoplasmic reticulum membrane. Proc Natl Acad Sci USA, 2005, 102: 14296-14301

28 Tavaria M, Gabriele T, Anderson R L, et al. Localization of the gene encoding the human heat shock cognate protein, HSP73, to chromosome 11. Genomics, 1995, 29: 266-268

29 Yang Y, Turner R S, Gaut J R. The chaperone BiP/GRP78 binds to amyloid precursor protein and decreases Abeta40 and Abeta42 secretion. J Biol Chem, 1998, 273: 25552-25555

30 Heymann J B, Iwasaki K, Yim Y I, et al. Visualization of the binding of Hsc70 ATPase to clathrin baskets: Implications for an uncoating mechanism. J Biol Chem, 2005, 280: 7156-7161

31 Rapoport I, Boll W, Yu A, et al. A motif in the clathrin heavy chain required for the Hsc70/auxilin uncoating reaction. Mol Biol Cell, 2008, 19: 405-413

32 Pleasure I T, Black M M, Keen J H. Valosin-containing protein, VCP, is a ubiquitous clathrin-binding protein. Nature, 1993, 365: 459-462

33 Shi J, Dixon R A, Gonzales R A, et al. Identification of cDNA clones encoding valosin-containing protein and other plant plasma membrane-associated proteins by a general immunoscreening strategy. Proc Natl Acad Sci USA, 1995, 92: 4457-4461

34 Nagahama M, Suzuki M, Hamada Y, et al. SVIP is a novel $\mathrm{VCP} / \mathrm{p} 97-$ interacting protein whose expression causes cell vacuolation. Mol Biol Cell, 2003, 14: 262-273

35 Heo J M, Livnat-Levanon N, Taylor E B, et al. A stress-responsive system for mitochondrial protein degradation. Mol Cell, 2010, 40: 465-480

36 Tresse E, Salomons F A, Vesa J, et al. VCP/p97 is essential for maturation of ubiquitin-containing autophagosomes and this function is impaired by mutations that cause IBMPFD. Autophagy, 2010, 6: 217-227

37 Cunningham P N, Hack B K, Ren G, et al. Glomerular complement regulation is overwhelmed in passive Heymann nephritis. Kidney Int, 2001, 60: 900-909

38 Glassock R J. The pathogenesis of idiopathic membranous nephropathy: A 50-year odyssey. Am J Kidney Dis, 2010, 56: 157-167

39 Inagi R, Nangaku M, Onogi H, et al. Involvement of endoplasmic reticulum (ER) stress in podocyte injury induced by excessive protein accumulation. Kidney Int, 2005, 68: 2639-2650

40 He F, Chen S, Wang H, et al. Regulation of CD2-associated protein influences podocyte endoplasmic reticulum stress-mediated apoptosis induced by albumin overload. Gene, 2011, 484: 18-25

41 Lai M T, Huang K L, Chang W M, et al. Geldanamycin induction of grp78 requires activation of reactive oxygen species via ER stress responsive elements in 9L rat brain tumour cells. Cell Signal, 2003, 15: 585-595

42 Matthews J A, Belof J L, Acevedo-Duncan M, et al. Glucosamineinduced increase in Akt phosphorylation corresponds to increased endoplasmic reticulum stress in astroglial cells. Mol Cell Biochem, 2007, 298: 109-123

43 Kammoun H L, Chabanon H, Hainault I, et al. GRP78 expression inhibits insulin and ER stress-induced SREBP-1c activation and reduces hepatic steatosis in mice. J Clin Invest, 2009, 119: 1201-1215

44 Vij N, Fang S, Zeitlin P L. Selective inhibition of endoplasmic reticulum-associated degradation rescues DeltaF508-cystic fibrosis transmembrane regulator and suppresses interleukin-8 levels: Therapeutic implications. J Biol Chem, 2006, 281: 17369-17378

45 Lass A, McConnell E, Nowis D, et al. A novel function of VCP (valosin-containing protein; p97) in the control of $N$-glycosylation of proteins in the endoplasmic reticulum. Arch Biochem Biophys, 2007, 462: 62-73

46 Zhong X, Shen Y, Ballar P, et al. AAA ATPase p97/valosin-containing 
protein interacts with gp78, a ubiquitin ligase for endoplasmic reticulum-associated degradation. J Biol Chem, 2004, 279: 45676-45684

47 Wojcik C, Rowicka M, Kudlicki A, et al. Valosin-containing protein (p97) is a regulator of endoplasmic reticulum stress and of the degradation of $\mathrm{N}$-end rule and ubiquitin-fusion degradation pathway substrates in mammalian cells. Mol Biol Cell, 2006, 17: 4606-4618

Open Access This article is distributed under the terms of the Creative Commons Attribution License which permits any use, distribution, and reproduction in any medium, provided the original author(s) and source are credited.

\section{Supporting Information}

Figure S1 Protein-GO networks of all proteins.

The supporting information is available online at csb.scichina.com and www.springerlink.com. The supporting materials are published as submitted, without typesetting or editing. The responsibility for scientific accuracy and content remains entirely with the authors. 